\title{
Polydatin inhibits hepatocellular carcinoma via the AKT/STAT3-FOXO1 signaling pathway
}

\author{
JIAN JIANG $^{1 *}$, YAODONG CHEN ${ }^{1 *}$, TIANXIU DONG ${ }^{1}$, MINLU YUE $^{1}$, YU ZHANG $^{1}$, \\ TINGTING AN ${ }^{1}$, JIUWEI ZHANG ${ }^{1}$, PENGFEI LIU ${ }^{2}$ and XIUHUA YANG ${ }^{1}$ \\ Departments of ${ }^{1}$ Abdominal Ultrasonography and ${ }^{2}$ Magnetic Resonance Imaging, \\ The First Affiliated Hospital of Harbin Medical University, Harbin, Heilongjiang 150001, P.R. China
}

Received April 11, 2018; Accepted January 15, 2019

DOI: $10.3892 / \mathrm{ol} .2019 .10123$

\begin{abstract}
Polydatin, extracted from Polygonum cuspidatum, is known for its anti-platelet aggregation and anti-inflammatory effects. However,studies on the association of polydatin with cancer are limited, particularly with regards to epithelial-mesenchymal transition (EMT)-associated migration and invasion of cancer cells. The purpose of the present study was to reveal the potential anticancer effects of polydatin on hepatocellular carcinoma (HCC) cells, particularly its effects on EMT. MTT assay was used to determine cell viability. Migration and invasion were evaluated through wound healing and transwell assays. Colony formation efficiency assay was conducted to detect proliferation. Flow cytometric analyses of apoptosis and cell cycle progression were performed following cells staining with Annexin V-fluorescein isothiocyanate (FITC)/propidium iodide (PI) and PI alone, respectively. Western blotting was used to investigate relevant molecular mechanisms. The results indicated that polydatin inhibited proliferation via $\mathrm{G}_{2} / \mathrm{M}$ arrest, suppressed migration and invasion of HCC cells, and promoted their apoptosis. In addition, phosphorylated (p)-protein kinase B (AKT), p-Janus kinase 1 and p-signal transducer and activator of transcription 3 (STAT3) levels were decreased as polydatin concentrations increased, and forkhead box protein O1 (FOXO1) expression was upregulated. Furthermore, the expression levels of various markers of EMT were reversed following treatment with polydatin. In conclusion, the present study validated that polydatin may inhibit proliferation via $\mathrm{G}_{2} / \mathrm{M}$ arrest, and suppressed EMT-associated migration and invasion of HCC cells. The results also suggested
\end{abstract}

Correspondence to: Professor Pengfei Liu, Department of Magnetic Resonance Imaging, The First Affiliated Hospital of Harbin Medical University, 23 Youzheng Street, Harbin, Heilongjiang 150001, P.R. China

E-mail: liup.fei@163.com

*Contributed equally

Key words: polydatin, hepatocellular carcinoma, protein kinase B/ signal transducer and activator of transcription 3-forkhead box protein $\mathrm{O} 1$, epithelial-mesenchymal transition, migration and invasion that polydatin may promote HCC cell apoptosis by blocking the AKT/STAT3-FOXO1 signaling pathway.

\section{Introduction}

Hepatocellular carcinoma (HCC) is a common primary liver malignancy, which represents the fifth and seventh most common type of cancer worldwide in men and women, respectively (1). The malignancy of HCC is attributed to its fast progression, the rapid development of metastasis and the absence of efficient curative therapy, all leading to an unfavorable prognosis (2). Treatments available for HCC include surgical resection, regional ablation and liver transplantation; however, these options are not proposed to patients diagnosed at an advanced stage. Treatment with near infrared-induced hyperthermia exerts promising inhibitory effects on $\mathrm{HCC}$, although the poor penetration of the light restricts its application (3). In addition, numerous patients with HCC present with tolerance to sorafenib, which is a common United States Food and Drug Administration-approved chemotherapeutic drug for patients at the advanced stage (4). Additionally, some novel tyrosine kinase inhibitors, including lenvatinib, cabozantinib and regorafenib, have demonstrated promising therapeutic effects in recent clinical trials. However, the efficacy, side effects and toxicity associated with these treatments still require further study (5-7). It is therefore crucial to explore novel anti-HCC drugs and therapeutic approaches.

Activation of epithelial-mesenchymal transition (EMT) is characterized by the progression of an epithelial phenotype to a mesenchymal phenotype. It is a crucial event for tumor metastasis, particularly in the early stages of disease (8). In addition, numerous EMT-related genes are associated with metastasis and the recurrence of cancer (9-11). In HCC, EMT is regulated by various oncogenes and tumor suppressor genes, and stimulates venous invasion and metastasis, leading to poor prognosis (12). Forkhead box O1 (FOXO1) belongs to the forkhead family of transcription factors characterized by a distinct forkhead domain. FOXO1 is considered a tumor suppressor gene that is regulated by the phosphoinositide 3-kinase (PI3K)/protein kinase B (AKT) and Janus kinase 1 (JAK1)/signal transducer and activator of transcription 3 (STAT3) signaling pathways, forming the AKT/STAT3-FOXO1 signaling pathway $(13,14)$. Our previous studies have revealed that FOXO1 inhibits the invasion and metastasis of HCC by reversing zinc finger 
E-box-binding homeobox 2-induced EMT (15). Furthermore, various overexpressed oncogenes in $\mathrm{HCC}$, including Epidermal growth factor receptor kinase substrate 8-like protein 3 and zinc finger and BTB domain-containing 20, are able to promote proliferation and inhibit apoptosis of $\mathrm{HCC}$ by repressing FOXO1, suggesting that FOXO1 may inhibit proliferation and stimulate apoptosis $(16,17)$. These findings suggested that the AKT/STAT3-FOXO1 signaling pathway may participate in several processes involved in the progression of HCC, including proliferation, apoptosis, and EMT-associated migration and invasion.

Polydatin, also named pieceid, (E)-piceid, (E)-polydatin, trans-polydatin and 3,40,5-trihydroxystilbene-3-b-D-glucoside, is a monocrystalline compound originally extracted from the root and rhizome of Polygonum cuspidatum (Fig. 1). Polydatin is also detectable in grapes, peanuts, hop cones, red wine, hop pellets, cocoa-containing products and chocolate. Previous studies have demonstrated that polydatin has many biological functions, including the prevention of platelet aggregation (18), and cardioprotective (19) and anti-inflammatory (20) properties. In addition, polydatin stimulates apoptosis and cell cycle arrest in lung and colorectal cancers $(21,22)$. However, the effects of polydatin on HCC are currently unknown.

To the best of our knowledge, the present study suggested for the first time that polydatin may inhibit EMT-related migration and invasion, and proliferation (via $\mathrm{G}_{2} / \mathrm{M}$ arrest) of HCC cells, and may promote HCC cell apoptosis by limiting the AKT/STAT3-FOXO1 signaling pathway.

\section{Materials and methods}

Cells and reagents. The HCCLM3 human HCC cell line and LO2 normal hepatic cell line were purchased from the American Type Culture Collection (Manassas, VA, USA), and were cultured in Dulbecco's modified Eagle's medium (DMEM) containing $10 \%$ fetal bovine serum, $100 \mathrm{IU} / \mathrm{ml}$ penicillin and $100 \mu \mathrm{g} / \mathrm{ml}$ streptomycin, which were all obtained from Gibco, Thermo Fisher Scientific, Inc. (Waltham, MA, USA). Cells were incubated at $37^{\circ} \mathrm{C}$ in a humidified incubator containing $5 \%$ $\mathrm{CO}_{2}$. Polydatin (cat. no. P109977) was purchased from Aladdin Industrial Corporation (Shanghai, China). A stock solution of $350 \mathrm{mmol} / \mathrm{l}$ polydatin was prepared in dimethyl sulfoxide (DMSO) and freshly diluted in medium prior to experiments. MTT assay (cat. no. M2128) was purchased from Sigma-Aldrich (Merck KGaA, Darmstadt, Germany) and fluorescein isothiocyanate (FITC)-conjugated Annexin V/propidium iodide (PI) apoptosis detection kit was provided by 4A Beijing Biotech Co., Ltd. (Beijing, China). Primary and secondary antibodies were purchased from Cell Signaling Technology, Inc. (Danvers, MA, USA). The Bio-Rad protein assay kit II was supplied by Bio-Rad Laboratories, Inc. (Hercules, CA, USA) and the enhanced chemiluminescent western blot detection reagents (cat. no. RPN2106) were obtained from GE Healthcare (Chicago, IL, USA).

Cytotoxicity assay. The cytotoxicity of polydatin was measured using the MTT assay. Cells were seeded in 96-well plates at $4 \times 10^{3}$ cells/well for $24 \mathrm{~h}$. HCCLM3 and LO2 cells were treated with increasing doses of polydatin $(0-800 \mu \mathrm{mol} / \mathrm{l})$ for various durations $(24-72 \mathrm{~h})$. MTT solution $(5 \mathrm{mg} / \mathrm{ml}$ in DMEM medium) was added (20 $\mu \mathrm{l} /$ well) and plates were further incubated for $4 \mathrm{~h}$ at $37^{\circ} \mathrm{C}$. A volume of $100 \mu \mathrm{l}$ DMSO was added to each well to solubilize the formazan product prior to measuring the absorbance with a microplate reader at $490 \mathrm{~nm}$. The assays were performed three times.

Colony formation efficiency assay. HCCLM3 cells were seeded in 6-well plates at a density of $1 \times 10^{3}$ cells/well for $24 \mathrm{~h}$. Culture medium was replaced with DMEM containing different doses of polydatin $(0-800 \mu \mathrm{mol} / \mathrm{l})$ and cells were incubated for $24 \mathrm{~h}$ at $37^{\circ} \mathrm{C}$. The supernatant was replaced with $2 \mathrm{ml}$ regular DMEM containing $10 \% \mathrm{FBS}$, and cells were cultured for 2 weeks at $37^{\circ} \mathrm{C}$ until visible cell clones were formed. Once colonies were formed, cells were fixed with $4 \%$ paraformaldehyde (PFA) for $25 \mathrm{~min}$ and washed three times with PBS at room temperature. Cells were stained with crystal violet for $25 \mathrm{~min}$ and rinsed three times with PBS at room temperature. Colonies were counted in a double blind manner using a light microscope (Shanghai CSOIF Co., Ltd., Shanghai, China). Results were presented as the percentage of colony numbers (average colony numbers of each group compared with control), and the assays were replicated three times.

Wound healing assay. HCCLM3 cells were seeded into 6 -well plates at $2.5 \times 10^{5}$ cells/well and cultured at $37^{\circ} \mathrm{C}$ until a monolayer was formed. Cells were scratched with a sterile micropipette tip and treated with $20 \mu \mathrm{g} / \mathrm{ml}$ mitomycin (Aladdin Industrial Corporation) for $20 \mathrm{~min}$. Cells were washed with PBS to remove debris, and further cultivated with serum-free medium containing different doses of polydatin $(0-150 \mu \mathrm{mol} / \mathrm{l})$ for 24 and $48 \mathrm{~h}$. The migration distance was measured and analyzed by Image J v1.8.0 (National Institutes of Health, Bethesda, MD, USA), and the assays were repeated three times.

Migration and invasion assays. Transwell chambers (pore size, $8 \mu \mathrm{m}$ ) were used to detect the migration and invasion of HCCLM3 cells pretreated with polydatin ( $0-150 \mu \mathrm{mol} / \mathrm{l})$ for $48 \mathrm{~h}$ at $37^{\circ} \mathrm{C}$. For the migration assay, $5 \times 10^{4}$ cells were seeded in serum-free DMEM $(200 \mu \mathrm{l})$ into the upper chambers and $500 \mu \mathrm{l}$ DMEM containing $10 \%$ FBS was added to the lower chamber. For the invasion assay, the upper polycarbonate membranes of the chambers were coated with $5 \mathrm{mg} / \mathrm{ml}$ Matrigel. Following a $48 \mathrm{~h}$ incubation, cells were fixed with $4 \%$ PFA for $20 \mathrm{~min}$ and stained with crystal violet for $20 \mathrm{~min}$. Cells in three randomly chosen fields were assessed under a light microscope (Shanghai CSOIF Co., Ltd). Each assay was performed three times.

Analysis of apoptosis. Annexin V-FITC/PI double staining was conducted to examine the apoptosis of HCCLM3 cells following treatment with polydatin. Briefly, cells were seeded in 6-well plates at a density of $2.5 \times 10^{5}$ cells/well and were cultured overnight to allow adhesion. Following treatment with increasing doses of polydatin $(0-800 \mu \mathrm{mol} / \mathrm{l})$ for $48 \mathrm{~h}$ at $37^{\circ} \mathrm{C}$, cells were stained with Annexin V-FITC/PI for $15 \mathrm{~min}$ in the dark, according to the manufacturer's protocol. Apoptotic cells were detected by flow cytometry using the FACSCalibur flow cytometer (BD Biosciences, Franklin Lakes, NJ, USA). The assays were carried out three times.

Cell cycle analysis. HCCLM3 cells were seeded in 6-well plates $\left(2.5 \times 10^{5} /\right.$ well $)$. At $24 \mathrm{~h}$, cells were treated with 
<smiles>OC[C@H]1O[C@@H](Oc2cc(O)cc(/C=C/c3ccc(O)cc3)c2)[C@H](O)[C@@H](O)[C@@H]1O</smiles>

Figure 1. Chemical structure of polydatin.

$200 \mu \mathrm{mol} / 1$ polydatin for an additional $48 \mathrm{~h}$ at $37^{\circ} \mathrm{C}$. Following trypsinization, cells were washed with PBS and fixed with $75 \%$ ethanol for $4 \mathrm{~h}$ at $4^{\circ} \mathrm{C}$. Cells were washed twice with PBS and covered with $0.5 \mathrm{ml}$ PBS containing $20 \mu \mathrm{l}$ RNaseA and PI for $30 \mathrm{~min}$ at $4^{\circ} \mathrm{C}$. The cell cycle progression of HCC cells was detected using the FACSCalibur flow cytometer with an excitation wavelength of $488 \mathrm{~nm}$, and emission wavelength of at $670 \mathrm{~nm}$. Cell cycle progression was analyzed using ModFit LT 4.1 (Verity Software House, Topsham, ME, USA) by gating live cells.

Western blotting. HCCLM3 cells were seeded into 6-well plates at $2.5 \times 10^{5}$ cells/well and incubated at $37^{\circ} \mathrm{C}$ overnight. The supernatant was replaced by fresh medium containing increasing concentrations of polydatin (0-800 $\mu \mathrm{mol} / \mathrm{l})$ for $48 \mathrm{~h}$ at $37^{\circ} \mathrm{C}$. Following treatment, cells were lysed with RIPA buffer (Aladdin Industrial Corporation) and subjected to protein extraction. Protein concentrations were determined using the Pierce Micro BCA protein assay system (Pierce; Thermo Fisher Scientific, Inc.). Equal amounts of protein $(25 \mu \mathrm{g})$ from each sample were separated via SDS-PAGE on a $10 \%$ gel, and transferred to nitrocellulose membranes that were further blocked with $5 \%$ non-fat milk (diluted in TBST containing $0.1 \%$ Tween-20) for $1 \mathrm{~h}$ at room temperature. Target proteins were immunoblotted with the appropriate primary and horseradish peroxidase (HRP)-conjugated secondary antibodies, which were all diluted at 1:1,000. The following antibodies were purchased from Wuhan Sanyang Biotechnology (Wuhan, China): Cyclin B1 (cat. no. 55004-1-AP) Cyclin D1 (cat. no. 60186-1-Ig) Bax (cat. no. 60267-1-Ig) Bcl-2 (cat. no. 60178-1-Ig) $\beta$-actin (cat. no. 20536-1-Ig) Vimentin (cat. no. 60,330-1-Ig) N-Cadherin (cat. no. 66219-1-Ig) E-Cadherin (cat. no. 60335-1-Ig), p-AKT (cat. no. 66444-1-Ig), AKT (cat. no. 60203-2-Ig) and FOXO1 (cat. no. 66457-1-Ig). The following antibodies were obtained from Abcam (Shanghai, China): p-JAK1 (cat. no. ab138005), JAK1 (cat. no. ab47435), p-STAT3 (cat. no. ab76315), STAT3 (cat. no. ab119352), p-p38 (cat. no. ab4822) and p38 (cat. no. ab31828). Primary antibodies were incubated at room temperature for $2 \mathrm{~h}$. Following washing the membranes with PBST containing $0.1 \%$ Tween-20 three times, the bound antibodies were then detected using the secondary goat anti-rabbit or goat anti-mouse antibodies for $1 \mathrm{~h}$ at room temperature and protein signals were visualized by enhanced chemiluminescence using ECL Western blotting detection reagents (Beyotime Institute of Biotechnology, Haimen, China) for $1 \mathrm{~min}$ and exposed to Kodak Biomax XAR film. The experiments were repeated three times.

Statistical analysis. All data were analyzed using SPSS 22.0 software (IBM Corp., Armonk, NY, USA). Results were expressed as the mean \pm standard deviation. One-way analysis of variance was used to compare two groups of data. Multiple comparisons between the groups were performed using the Student-Newman-Keuls method. $\mathrm{P}<0.05$ was considered to indicate a statistically significant difference.

\section{Results}

Cytotoxic effects of polydatin on HCCLM3 cells. The associations between drug cytotoxicity and dose/time-dependent drug efficacy were the first parameters considered. In addition, an absence of toxicity of chemotherapeutic drugs on human normal cells is essential when developing a novel treatment, particularly for liver cancer, since the liver is the major organ involved in drug metabolism.

Results revealed that polydatin was not toxic for LO2 cells (Fig. 2A). Furthermore, polydatin was significantly toxic to HCCML3 cells, at concentrations of 300 and $200 \mu \mathrm{mol} / 1$ after 24 and $48 \mathrm{~h}$, respectively. However, concentrations $<200 \mu \mathrm{mol} / 1 \mathrm{did}$ not exhibit any cytotoxicity for all treatment durations. Furthermore, for concentrations $>200 \mu \mathrm{mol} / 1$, polydatin displayed toxicity in a dose-dependent manner. In combination with the drug half maximal inhibiting concentration illustrated in Fig. 2B, these results suggested that polydatin may effectively destroy HCC cells with low toxicity in healthy human hepatic cells.

Effects of polydatin on HCCLM3 cell migration. Wound healing and transwell chamber assays were performed to investigate the effects of polydatin on the migration of HCC cells. HCCLM3 cells were cultured with nontoxic doses of polydatin $(0-150 \mu \mathrm{mol} / \mathrm{l})$ to avoid interference with cell toxicity. Fig. 3A demonstrated that polydatin decreased the wound healing of HCCLM3 cells in a dose-dependent manner. In comparison with control cells, the relative migration level of HCCLM3 cells treated with $150 \mu \mathrm{mol} / 1$ polydatin was $\sim 0.27$-fold at $48 \mathrm{~h}$ (Fig. 3B). In addition, the migration assay demonstrated that migrated cells on the lower side of the membrane were reduced in a dose-dependent manner (Fig. 3C and D), which confirmed the results of the wound healing assay. These data suggested that polydatin may prevent HCCML3 migration in a dose-dependent manner.

Effects of polydatin on HCCLM3 cell invasion. Compared with cell migration, which only involves mobility, cell invasion also involves the ability of cells to degrade the extracellular matrix. Therefore, Transwell chambers covered with Matrigel were used to detect the effects of polydatin on HCC cell invasion. Fig. 4A and B revealed that the number of invasive cells was significantly decreased following polydatin treatment in a dose-dependent manner. In comparison with control cells, the relative invasion of HCCLM3 cells treated with $150 \mu \mathrm{mol} / 1$ polydatin was $\sim 0.09$-fold. These findings suggested that polydatin may inhibit the invasive ability of HCCLM3 cells.

Polydatin inhibits EMT in HCCLM3 cells. EMT is a crucial process for migration and invasion of cancer cells. In the present study, polydatin was suspected to suppress migration and invasion of HCCLM3 cells by inhibiting EMT. To further 

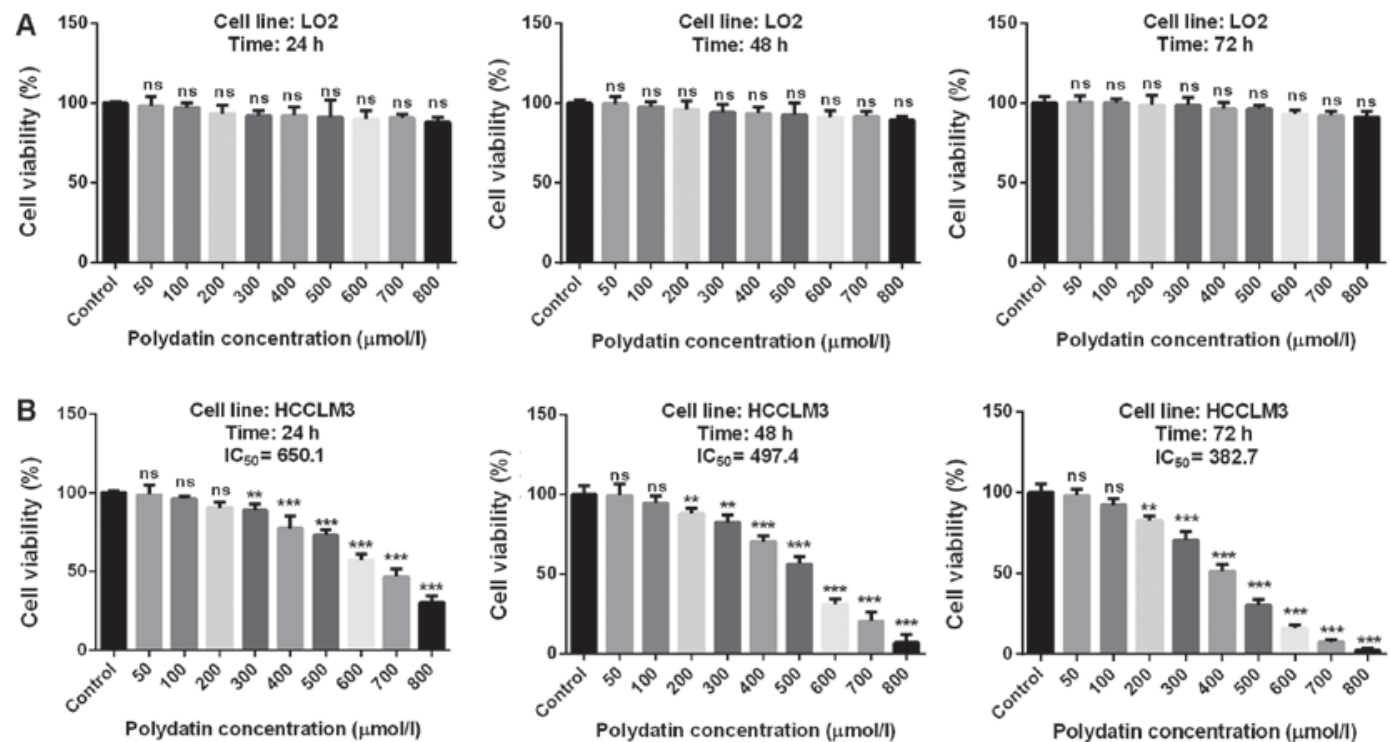

Figure 2. Effects of polydatin on cell viability of LO2 and HCCLM3 cells. Cytotoxicity of polydatin at various concentrations and treatment durations in (A) LO2 and (B) HCCLM3 cells. ${ }^{* *} \mathrm{P}<0.01,{ }^{* * *} \mathrm{P}<0.001$ vs. control. $\mathrm{IC}_{50}$, half maximal inhibitory concentration; ns, not significant.

A

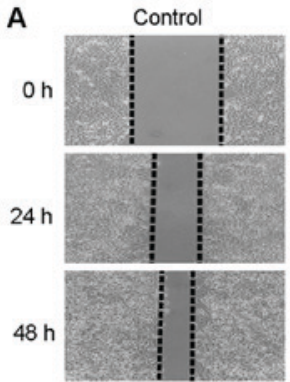

C

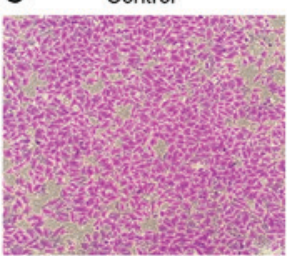

$100 \mu \mathrm{mol} / \mathrm{l}$

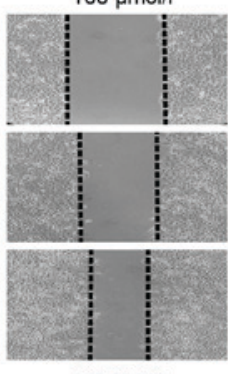

HCCLM3

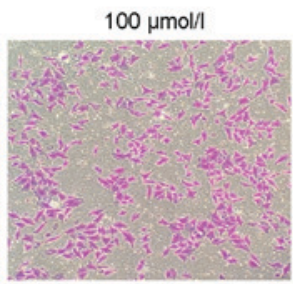

HCCLM3

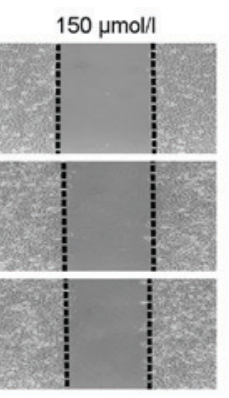

$150 \mu \mathrm{mol} / \mathrm{I}$

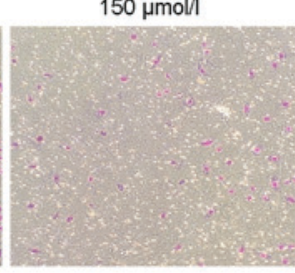

B
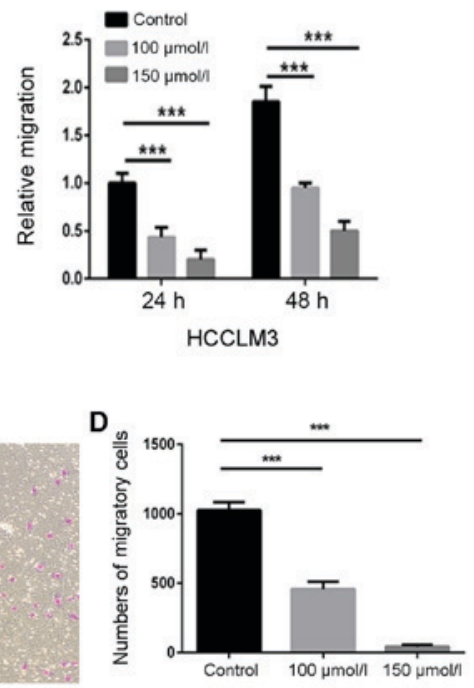

HCCLM3

Figure 3. Polydatin inhibits migration of HCCLM3 cells. (A) Suppressive effects of polydatin on migration were assessed via wound healing assay (magnification, x200). (B) Statistical analysis of the relative distances of cell migration (C) Migration assay of HCCLM3 cells following treatment with polydatin at non-cytotoxic concentrations $(0-150 \mu \mathrm{mol} / 1)$ (magnification, $\mathrm{x} 200)$. (D) Statistical analysis of the number of cells crossing the membrane. ${ }^{* * *} \mathrm{P}<0.001$ vs. control.

investigate the effects of polydatin on EMT, western blotting was used to detect the EMT-associated markers in HCCLM3 cells following treatment with polydatin for $48 \mathrm{~h}$. The results suggested that the expression levels of the mesenchymal markers $\mathrm{N}$-cadherin and vimentin were decreased by polydatin in a dose-dependent manner, whereas the expression levels of the epithelial marker E-cadherin were increased (Fig. 5A and B). These findings suggested that polydatin may prevent migration and invasion of HCCLM3 cells by reversing the EMT process.

Anti-proliferative effects of polydatin on HCCLM3 cells. Proliferation is an essential process for tumorigenesis and development. In the present study, the colony formation assay was performed to evaluate the proliferative ability of HCCLM3 cells following treatment with polydatin. The results demonstrated that polydatin significantly inhibited HCCLM3 cell proliferation in a dose-dependent manner (Fig. 6A and B). It is generally accepted that cell proliferation is controlled by the cell cycle. The effect of polydatin on cell cycle progression was therefore assessed. As presented in Fig. 6C and D, polydatin arrested the HCCLM3 cell cycle at the $G_{2} / M$ phase. To further confirm this result, the expression levels of the proteins cyclin D1 and B1, which regulate the $G_{0} / G_{1}$ and $G_{2} / M$ phases, respectively, were analyzed by western blotting. The results revealed that the expression levels of cyclin B1 were markedly increased following polydatin treatment, whereas a decreased 
A

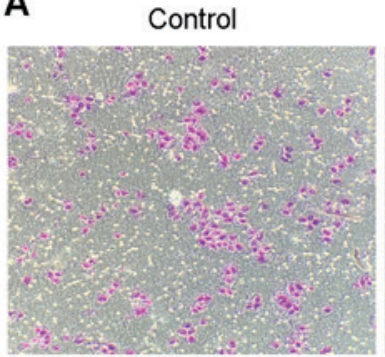

$100 \mu \mathrm{mol} / \mathrm{l}$

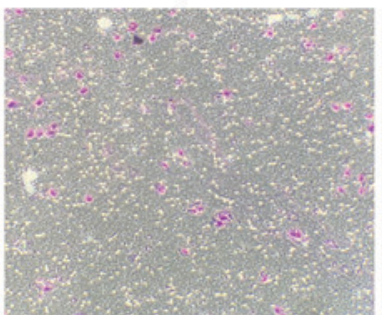

HCCLM3
$150 \mu \mathrm{mol} / \mathrm{l}$

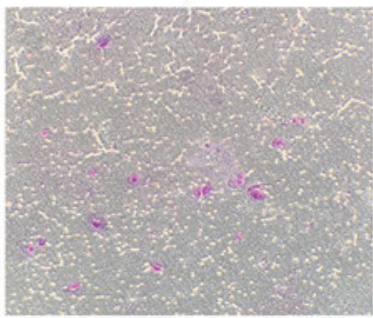

B

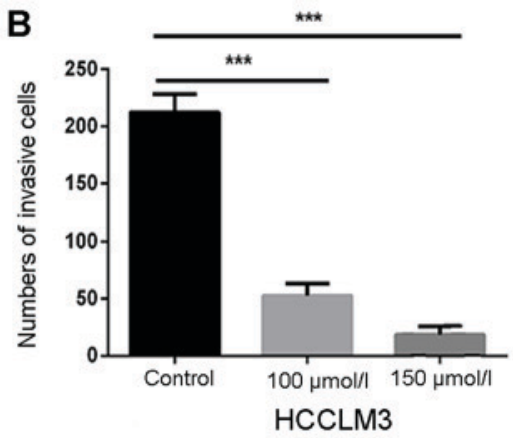

Figure 4. Polydatin suppresses invasion of HCCLM3 cells. (A) Invasion assays of HCCLM3 cells following treatment with polydatin at non-cytotoxic concentrations $(0-150 \mu \mathrm{mol} / \mathrm{l})$ (magnification, $\mathrm{x} 200)$. (B) Statistical analysis of number of cells crossing the membrane ${ }^{* * *} \mathrm{P}<0.001$ vs. control.

A

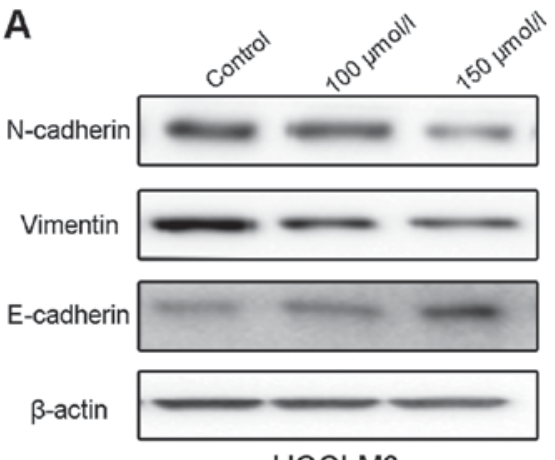

HCCLM3
B

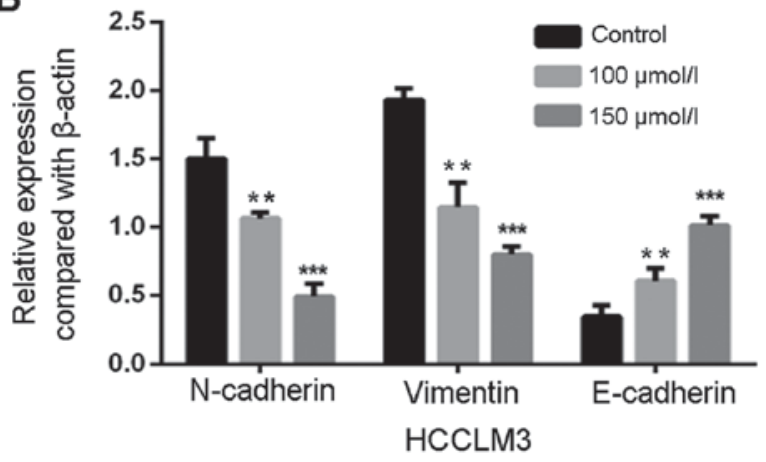

Figure 5. Polydatin represses epithelial-mesenchymal transition in HCCLM3 cells. (A) Protein expression of E-cadherin, N-cadherin and vimentin in HCCML3 cells following treatment with polydatin at non-toxic concentrations $(0-150 \mu \mathrm{mol} / \mathrm{l})$ evaluated by western blotting. (B) Statistical analysis of protein expression. ${ }^{* *} \mathrm{P}<0.01,{ }^{* * * *} \mathrm{P}<0.001$ vs. control.

A

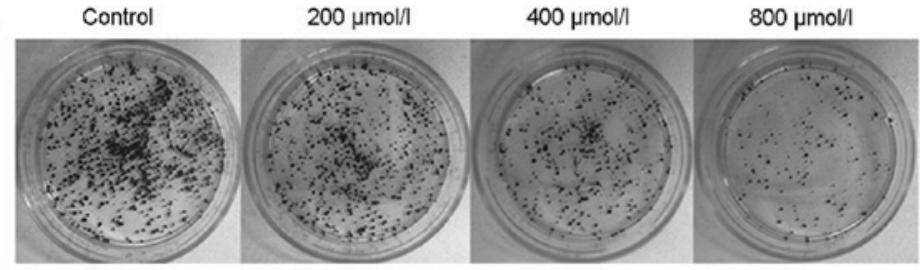

HCCLM3

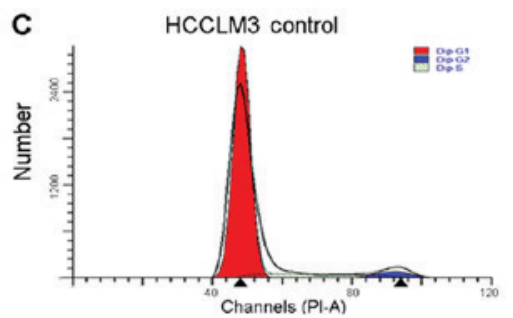

E

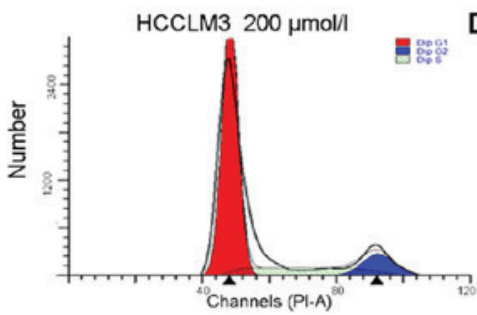

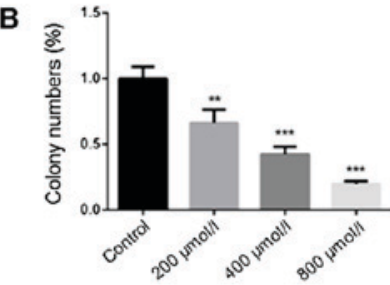

HCCLM3
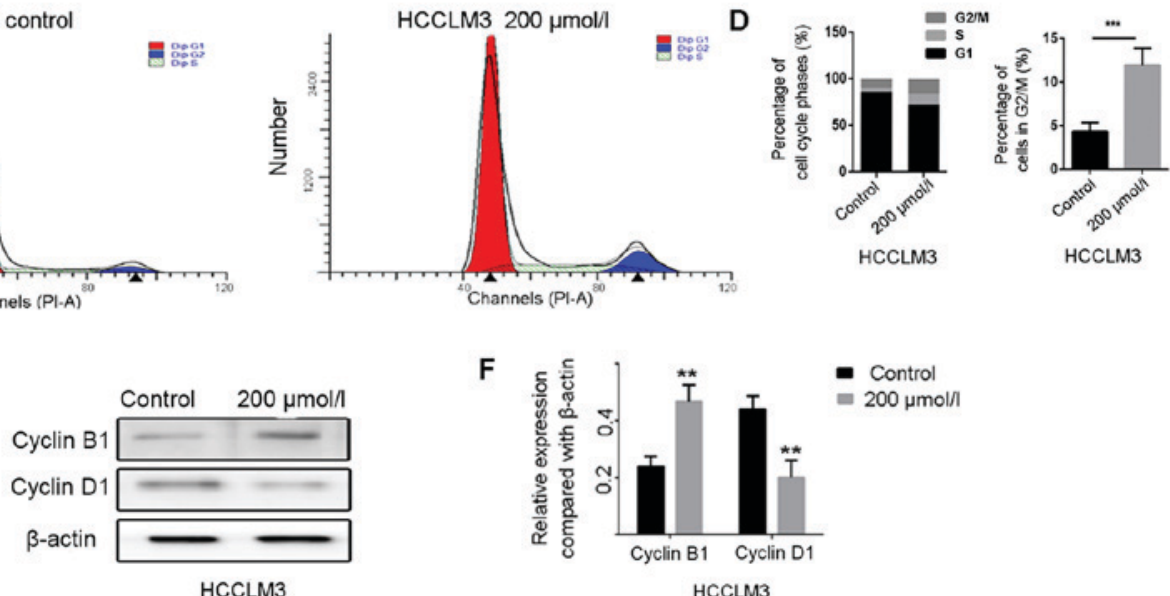

Figure 6. Polydatin inhibits proliferation of HCCLM3 cells. (A) Representative images of colony formation of HCCLM3 cells following treatment with polydatin at various concentrations. (B) Statistical analysis of the colony formation assay. (C) Cell cycle analysis results of HCCLM3 cells following treatment with or without $200 \mathrm{~mol} / \mathrm{l}$ polydatin. (D) Statistical analysis of the percentage of cells in the $\mathrm{G}_{2} / \mathrm{M}$ phase. (E) Alterations in protein expression levels of cyclin B1 and cyclin D1 in HCCML3 cells, following treatment with or without polydatin ( $200 \mu \mathrm{mol} / \mathrm{l})$ evaluated by western blotting. (F) Statistical analysis of protein expression. ${ }^{* *} \mathrm{P}<0.01,{ }^{* * * *} \mathrm{P}<0.001$ vs. control. 

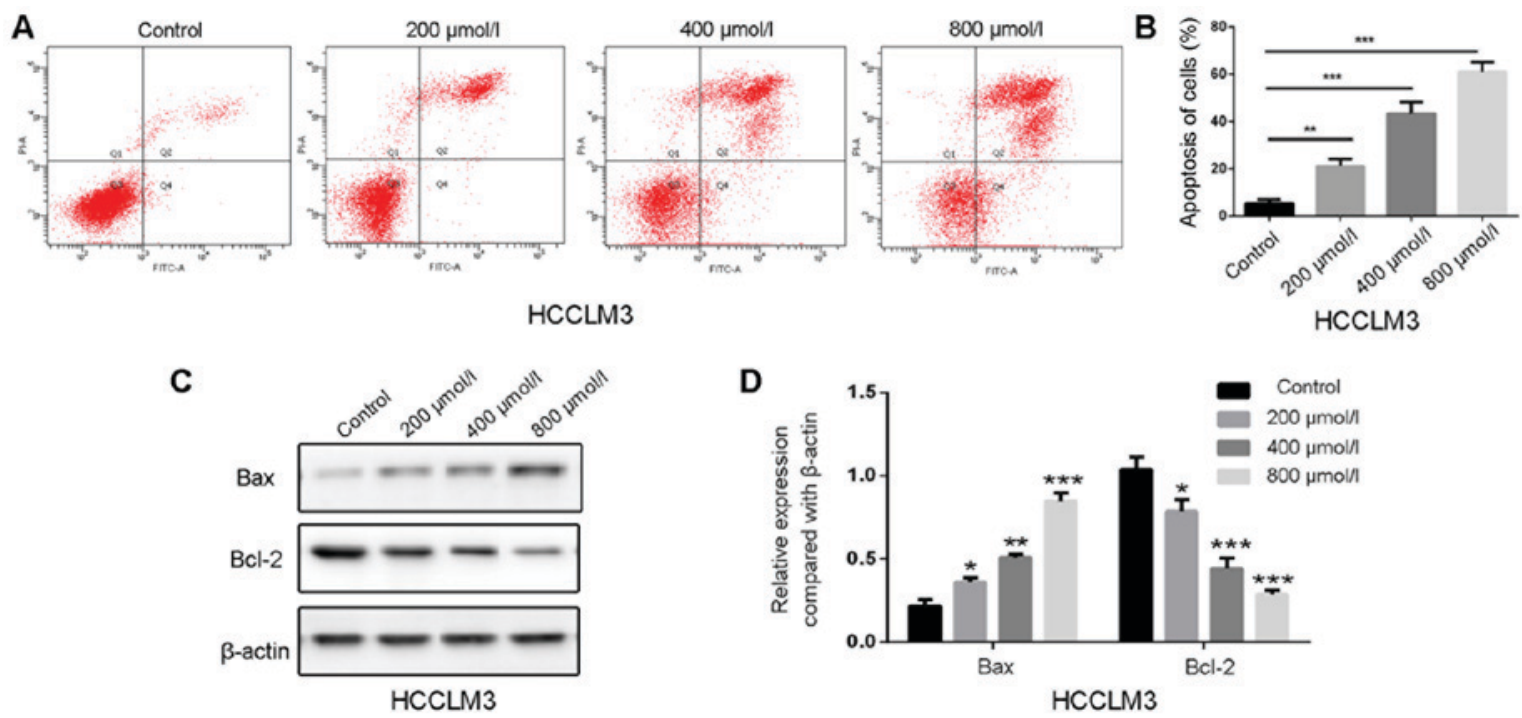

Figure 7. Polydatin induces apoptosis of HCCLM3 cells. (A) Apoptosis assays of HCCLM3 cells following treatment with polydatin at various concentrations. (B) Statistical analysis of cell apoptosis. (C) Changes in the protein expression levels of Bax and Bcl-2 in HCCML3 cells following treatment with polydatin at different concentrations evaluated by western blotting. (D) Statistical analysis of protein expression. ${ }^{*} \mathrm{P}<0.05$, ${ }^{* *} \mathrm{P}<0.01,{ }^{* * *} \mathrm{P}<0.001 \mathrm{vs}$. control. Bax, Bcl-2-associated X; Bcl-2, B-cell lymphoma 2.

expression of cyclin D1 was observed (Fig. 6E and F). These results suggested that polydatin may arrest the cell cycle at the $\mathrm{G}_{2} / \mathrm{M}$ phase, combined with a significantly reduced proportion of HCCML3 cells in the $G_{0} / G_{1}$ phase. Taken together, these data suggested that polydatin may inhibit HCCLM3 cell proliferation by inducing cell cycle arrest at the $\mathrm{G}_{2} / \mathrm{M}$ phase.

Polydatin induces apoptosis in HCCLM3 cells. Apoptosis is a primary type of cell death induced by chemotherapeutic drugs, and the effects of polydatin on apoptosis have been reported in human lung cancer cells (21). The effects of $48 \mathrm{~h}$ treatment of polydatin on HCCML3 cell apoptosis were therefore evaluated. As shown in Fig. 7A, polydatin induced HCCML3 cell apoptosis in a dose-dependent manner. At a concentration of $800 \mu \mathrm{mol} / 1$, the percentage of apoptotic cells reached $\sim 60 \%$ compared with untreated cells (Fig. 7B).

Apoptosis is mediated by death receptors, mitochondria and the endoplasmic reticulum (23). In the present study, the expression levels of the pro-apoptotic factor B-cell lymphoma 2 (Bcl-2)-associated $\mathrm{X}$ protein (Bax) increased following polydatin treatment, whereas the expression levels of the anti-apoptotic factor Bcl-2 were decreased (Fig. 7C and D). These results suggested that polydatin may induce HCCML3 cell apoptosis by regulating the expression of Bax and Bcl-2 in a dose-dependent manner.

Polydatin inhibits HCC via the AKT/STAT3-FOXOI signaling pathway. Results from the present study demonstrated that polydatin affected proliferation, apoptosis and EMT-associated migration and invasion in HCCLM3 cells. The possible underlying mechanism involved in these processes were therefore further investigated. Numerous signaling pathways, including PI3K/AKT, JAK1/STAT3 and p38, are known to serve important roles in the progression of HCC, including the EMT process (24-27). The effect of polydatin on these signaling pathways was thus evaluated. Following treatment with increasing doses of polydatin for
$48 \mathrm{~h}$, the expression levels of the central proteins involved in the aforementioned pathways were evaluated in HCCLM3 cells by western blotting. The following were detected: Activated AKT [phosphorylated (p)-AKT, Ser 473], AKT, activated JAK1 (p-JAK1, Tyr 1034/1035), JAK1, activated STAT3 (p-STAT3, Tyr 705), STAT3, activated p38 (p-p38, Thr 180/Tyr 182) and p38. The results demonstrated that polydatin induced a downregulation of p-AKT, p-JAK1 and p-STAT3 as compared with the control group; however, no significant changes were observed in p-p38 levels (Fig. 8A and B). These results suggested that polydatin may suppress $\mathrm{HCC}$ via the regulation of the PI3K/AKT and JAK1/STAT3 signaling pathways. As a downstream gene of these two signaling pathways, FOXO1 has garnered interest for its involvement in EMT, in addition to its well-known involvement in cell proliferation and apoptosis. The expression of FOXO1 was therefore evaluated. The results revealed that following treatment of HHCML3 cells with polydatin, the expression levels of FOXO1 were upregulated in a dose-dependent manner (Fig. 8A and B). These results suggested that polydatin may exert anti-HCC effects via the AKT/STAT3-FOXO1 signaling pathway.

\section{Discussion}

Natural herbal medicines have gained increasing attention in the development of chemotherapeutic regimens. Polydatin is a glycoside of resveratrol, in which the glycoside group is bound in the $\mathrm{C}-3$ position, leading to alterations in its biological properties. Compared with resveratrol, polydatin is more efficiently absorbed by the digestive system via an active mechanism using glucose carriers (28-30). Polydatin is also more resistant to enzymatic oxidation. In addition, polydatin preserves the active groups of resveratrol, including the two benzene rings with a hydroxyl group and double bonds between the two rings, whose anticancerous properties have previously been demonstrated $(31,32)$. Polydatin may therefore have some anticancer 

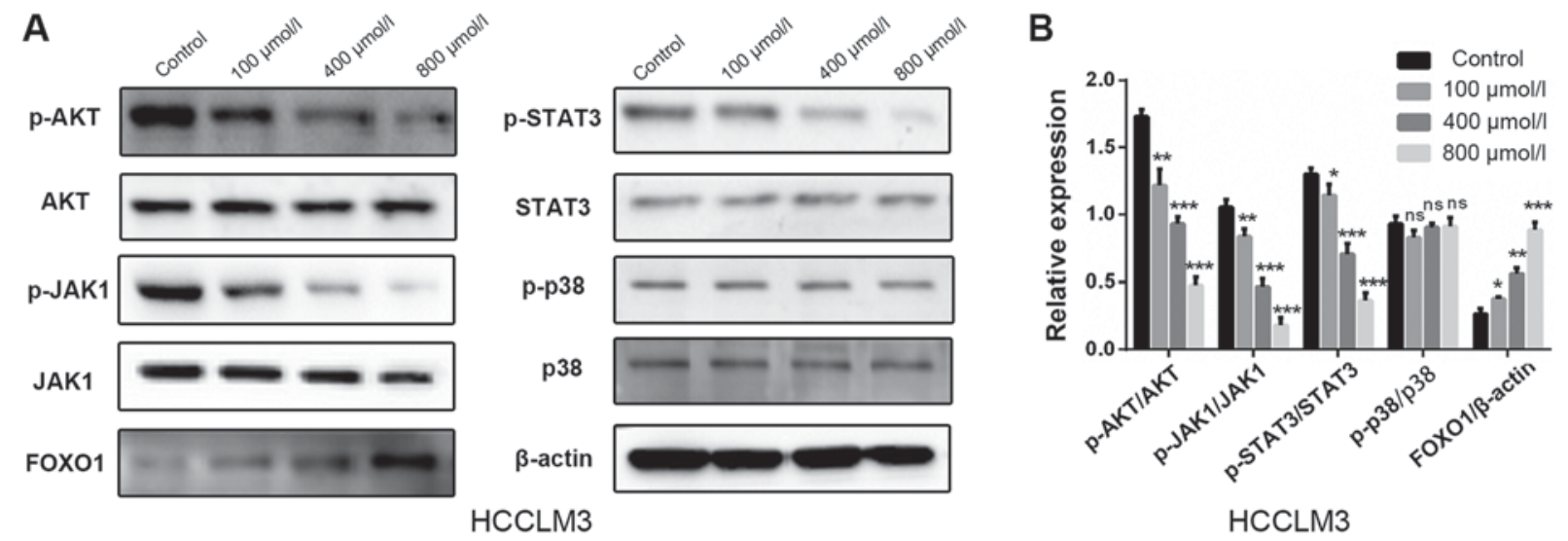

Figure 8. Polydatin inhibits hepatocellular carcinoma via the AKT/STAT3-FOXO1 signaling pathway. (A) Alterations in the expression levels of proteins involved in common signaling pathways in HCCML3 cells following treatment with polydatin at various concentrations, were evaluated by western blotting. (B) Statistical analysis of protein expression. All protein expression levels have been normalized to $\beta$-actin prior to further normalization with total protein level. ${ }^{*} \mathrm{P}<0.05,{ }^{* *} \mathrm{P}<0.01,{ }^{* * *} \mathrm{P}<0.001$ vs. control. AKT, protein kinase B; FOXO1, forkhead box protein O1; ns, not significant; STAT3, signal transducer and activator of transcription 3 .

potential. To the best of our knowledge, studies investigating the anticancer potential of polydatin are lacking $(21,22)$, with none suggesting an anti-EMT effect on HCC cells.

Tumor metastasis is a characteristic feature of malignant tumors, which is primarily responsible for cancer-associated mortality. Migration and invasion are essential conditions for tumor metastasis. In the present study, polydatin was demonstrated to inhibit the migration and invasion of HCCLM3 cells in a dose-dependent manner. In order to evaluate the anti-HCC effects of polydatin, numerous proliferation-associated experiments were performed, including colony formation efficiency, cell cycle analysis and apoptosis analysis. Results revealed that polydatin significantly inhibited proliferation and promoted apoptosis of HCCML3 cells in a dose-dependent manner.

Previous studies have revealed that EMT leads to increased cell migration and invasion in various types of cancer $(33,34)$. Subsequently, the present study further explored the influences of polydatin on EMT. The results revealed that treatment with polydatin increased the expression levels of E-cadherin, and decreased expression levels of $\mathrm{N}$-cadherin and vimentin. These results suggested that polydatin may promote EMT in HCCML3 cells in order to block their migration and invasion.

Three common signaling pathways have proven critical in the progression of HCC, including PI3K/AKT, JAK1/STAT3 and p38, and are closely associated with EMT in cancer metastasis (35). These pathways were therefore evaluated in the present study in HCCML3 cells following treatment with polydatin. The activation of AKT leads to the loss of tumor cell-cell junctions, disruption of tumor cell polarity and morphological alterations of tumor cells, thus stimulating tumor cell motility $(36,37)$. Furthermore, activated AKT inhibits the transcription of E-cadherin (24). Similarly, activation of STAT3 increases the mesenchymal level in various types of cancer cells $(25,26)$, whereas p38 participates in EMT in colorectal cancer progression (27). The results of the present study indicated that the levels of phosphorylated AKT, JAK1 and STAT3 were markedly decreased following treatment of HCCML3 cells with polydatin; however, no change was observed in p38 and p-p38 expression. The protein p38 is involved in oxidative stress regulation, suggesting that polydatin may not increase intracellular reactive oxygen species, which is consistent with the findings of previous studies $(38,39)$. FOXO1 belongs to the forkhead family and serves numerous roles, including cellular differentiation, proliferation, cell cycle progression, apoptosis and glucose metabolism (40). Previous studies have demonstrated that the expression levels of FOXO1 are closely associated with the EMT process in HCC $(15,41)$. By searching the Kyoto Encyclopedia of Genes and Genomes Pathway Database, FOXO1 was revealed to be a downstream gene of PI3K/AKT and JAK1/STAT3 signaling pathways $(42,43)$. This suggested that polydatin may control cell proliferation $\left(\mathrm{G}_{2} / \mathrm{M}\right.$ arrest $)$ and EMT-associated migration and invasion, and promote apoptosis of HCC cells via FOXO1 regulation. The expression of FOXO1 was therefore assessed and was increased in HCCLM3 cells following polydatin treatment. These results suggested that polydatin may serve therapeutic roles via increasing FOXO1 expression, which was regulated by PI3K/AKT and JAK1/STAT3 signaling pathways.

In conclusion, the present study demonstrated that polydatin may inhibit proliferation via $\mathrm{G}_{2} / \mathrm{M}$ arrest, suppress migration and invasion of HCC cells and promote HCC cell apoptosis in a dose dependent-manner. Overall, polydatin exhibited some therapeutic potential for HCC, and induced no toxicity in normal human cells. The study suggested that polydatin may exert therapeutic effects by inhibiting the AKT/STAT3-FOXO1 signaling pathway, and reducing proliferation, apoptosis and EMT-related migration and invasion. These findings may provide a novel theoretical foundation for the development of polydatin-based chemotherapeutic treatment for HCC.

\section{Acknowledgements}

Not applicable.

\section{Funding}

The present study was supported by the National Natural Science Foundation of China (grants no. 81771894) and the 
Fund of Scientific Research Innovation of The First Affiliated Hospital of Harbin Medical University (grant no. 2018B009).

\section{Availability of data and materials}

The datasets used and/or data analyzed during the present study are available from the corresponding author on reasonable request.

\section{Authors' contributions}

JJ, YC, PL and XY conceived this study. JJ and YC designed the experiments. JJ, YC, TD, MY and YZ performed the experiments. TA and JZ conducted the statistical analysis. JJ and YC wrote the manuscript. All authors read and approved the final manuscript.

\section{Ethics approval and consent to participate}

Not applicable.

\section{Patient consent for publication}

Not applicable.

\section{Competing interests}

The authors declare that they have no competing interests.

\section{References}

1. Heindryckx F, Colle I and Van Vlierberghe H: Experimental mouse models for hepatocellular carcinoma research. Int J Exp Pathol 90: 367-386, 2009.

2. Wei KR, Yu X, Zheng RS, Peng XB, Zhang SW, Ji MF, Liang ZH, Ou ZX and Chen WQ: Incidence and mortality of liver cancer in China, 2010. Chin J Cancer 33: 388-394, 2014.

3. Yang CY, Chen YD, Guo W, Gao Y, Song CQ, Zhang Q, Zheng NN, Han XJ and Guo CS: Bismuth ferrite-based nanoplatform design: An ablation mechanism study of solid tumor and NIR-triggered photothermal/photodynamic combination cancer therapy. Adv Funct Mater: 1706827, 2018.

4. Raza A and Sood GK: Hepatocellular carcinoma review: Current treatment, and evidencebased medicine. World $\mathrm{J}$ Gastroenterol 20: 4115-4127,2014.

5. Abou-Alfa GK, Meyer T, Cheng AL, El-Khoueiry AB, Rimassa L, Ryoo BY, Cicin I, Merle P, Park JW, Blanc JF, et al: Cabozantinib (C) versus placebo $(\mathrm{P})$ in patients (pts) with advanced hepatocellular carcinoma (HCC) who have received prior sorafenib: Results from the randomized phase III CELESTIAL trial. J Clin Oncol 36: 207, 2018

6. Bruix J, Qin S, Merle P, Granito A, Huang YH, Bodoky G, Pracht M, Yokosuka O, Rosmorduc O,Breder V, et al: Regorafenib for patients with hepatocellular carcinoma who progressed on sorafenib treatment (RESORCE): A randomised, double-blind, placebo-controlled, phase 3 trial. Lancet 389: 56-66, 2017.

7. Kudo M, Finn RS, Qin S, Han KH, Ikeda K, Piscaglia F, Baron A, Park JW, Han G, Jassem J, et al: Lenvatinib versus sorafenib in frst-line treatment of patients with unresectable hepatocellular carcinoma: A randomised phase 3 non-inferiority trial. Lancet 391: 1163-1173, 2018.

8. Puisieux A, Brabletz T and Caramel J: Oncogenic roles of EMT-inducing transcription factors. Nat Cell Biol 16: 488-494, 2014.

9. Yopp AC and Singal AG: Epithelial to mesenchymal transition expression profiles as predictive biomarkers of recurrence following resection of $\mathrm{HCC}$ : Implications for current clinical use and future stratification for systemic therapy. Ann Surg Oncol 21: 3723-3724, 2014.
10. Fransvea E, Angelotti U, Antonaci S and Giannelli G: Blocking transforming growth factor-beta up-regulates E-cadherin and reduces migration and invasion of hepatocellular carcinoma cells. Hepatology 47: 1557-1566, 2008.

11. Yamada S, Okumura N, Wei L, Fuchs BC, Fujii T, Sugimoto H, Nomoto S, Takeda S, Tanabe KK and Kodera Y: Epithelial to mesenchymal transition is associated with shorter disease-free survival in hepatocellular carcinoma. Ann Surg Oncol 21: 3882-3890, 2014

12. Wang CH, Guo ZY, Chen ZT, Zhi XT, Li DK, Dong ZR, Chen ZQ, Hu SY and Li T: TMPRSS4 facilitates epithelial-mesenchymal transition of hepatocellular carcinoma and is a predictive marker for poor prognosis of patients after curative resection. Sci Rep 5: 12366, 2015.

13. Boreddy SR, Pramanik KC and Srivastava SK: Pancreatic tumor suppression by benzyl isothiocyanate is associated with inhibition of PI3K/AKT/FOXO pathway. Clin Cancer Res 17: 1784-1795, 2011.

14. Aghazadeh S and Yazdanparast R: Mycophenolic acid potentiates HER2-overexpressing SKBR3 breast cancer cell line to induce apoptosis: Involvement of AKT/FOXO1 and JAK2/STAT3 pathways. Apoptosis 21: 1302-1314, 2016.

15. Dong T, Zhang Y, Chen Y, Liu P, An T, Zhang J, Yang H, Zhu W and Yang X: FOXO1 inhibits the invasion and metastasis of hepatocellular carcinoma by reversing ZEB2-induced epithelial-mesenchymal transition. Oncotarget 8: 1703-1713, 2017.

16. Zeng CX, Tang LY, Xie CY, Li FX, Zhao JY, Jiang N, Tong Z, $\mathrm{Fu}$ SB, Wen FJ and Feng WS: Overexpression of EPS8L3 promotes cell proliferation by inhibiting the transactivity of FOXO1 in HCC. Neoplasma 65: 701-707, 2018.

17. Kan H, Huang Y, Li X, Liu D, Chen J and Shu M: Zinc finger protein $\mathrm{ZBTB} 20$ is an independent prognostic marker and promotes tumor growth of human hepatocellular carcinoma by repressing FoxO1. Oncotarget 7: 14336-14349, 2016.

18. Liu LT, Guo G, Wu M and Zhang WG: The progress of the research on cardio-vascular effects and acting mechanism of polydatin. Chin J Integr Med 18: 714-719, 2012.

19. Gao JP, Chen CX, Gu WL, Wu Q, Wang Y and Lü J: Effects of polydatin on attenuating ventricular remodeling in isoproterenol-induced mouse and pressure-overload rat models. Fitoterapia 81: 953-960, 2010.

20. Shi YW, Wang CP, Liu L, Liu YL, Wang X, Hong Y, Li Z and Kong LD: Antihyperuricemic and nephroprotective effects of resveratrol and its analogues in hyperuricemic mice. Mol Nutr Food Res 56: 1433-1444, 2012.

21. Zhang Y, Zhuang Z, Meng Q, Jiao Y, Xu J and Fan S: Polydatin inhibits growth of lung cancer cells by inducing apoptosis and causing cell cycle arrest. Oncol Lett 7: 295-301, 2014.

22. De Maria S, Scognamiglio I, Lombardi A, Amodio N, Caraglia $\mathrm{M}$, Cartenì $\mathrm{M}$, Ravagnan $\mathrm{G}$ and Stiuso $\mathrm{P}$ : Polydatin, a natural precursor of resveratrol, induces cell cycle arrest and differentiation of human colorectal Caco-2 cell. J Transl Med 11: 264, 2013.

23. Redza-Dutordoir M and Averill-Bates DA: Activation of apoptosis signalling pathways by reactive oxygen species. Biochim Biophys Acta 1863: 2977-2992, 2016.

24. Larue L and Bellacosa A: Epithelial-mesenchymal transition in development and cancer: Role of phosphatidylinositol 3' kinase/AKT pathways. Oncogene 24: 7443-7454, 2005.

25. Chen YD, Zhang Y, Dong TX, Xu YT, Zhang W, An TT, Liu PF and Yang XH: Hyperthermia with different temperatures inhibits proliferation and promotes apoptosis through the EGFR/STAT3 pathway in C6 rat glioma cells. Mol Med Rep 16: 9401-9408, 2017.

26. Luwor RB, Baradaran B, Taylor LE, Iaria J, Nheu TV, Amiry N, Hovens CM, Wang B, Kaye AH and Zhu HJ: Targeting Stat3 and Smad7 to restore TGF- $\beta$ cytostatic regulation of tumor cells in vitro and in vivo. Oncogene 32: 2433-2441, 2013.

27. Wang B, Zhang L, Zhao L, Zhou R, Ding Y, Li G and Zhao L: LASP2 suppresses colorectal cancer progression through JNK/p38 MAPK pathway meditated epithelial-mesenchymal transition. Cell Commun Signal 15: 21, 2017.

28. Hollman PC, de Vries JH, van Leeuwen SD, Mengelers MJ and Katan MB: Absorption of dietary quercetin glycosides and quercetin in healthy ileostomy volunteers. Am J Clin Nutr 62: 1276-1282, 1995.

29. Paganga G and Rice-Evans CA: The identifcation of flavonoids as glycosides in human plasma. FEBS Lett 401: 78-82, 1997.

30. Krasnow MN and Murphy TM: Polyphenol glucosylating activity in cell suspensions of grape (Vitis vinifera). J Agric Food Chem 52: 3467-3472, 2004 
31. Delmas D, Aires V, Limagne E, Dutartre P, Mazué F, Ghiringhelli $\mathrm{F}$ and Latruffe N: Transport, stability, and biological activity of resveratrol. Ann N Y Acad Sci 1215: 48-59, 2011.

32. Chalal M, Klinguer A,Echairi A,Meunier P, Vervandier-FasseurD and Adrian M: Antimicrobial activity of resveratrol analogues. Molecules 19: 7679-7688, 2014.

33. Tsai JH and Yang J: Epithelial-mesenchymal plasticity in carcinoma metastasis. Genes Dev 27: 2192-2206, 2013.

34. Mitra A, Mishra L and Li S: EMT, CTCs and CSCs in tumor relapse and drug-resistance. Oncotarget 6: 10697-10711, 2015.

35. Hsu CY, Lin CH, Jan YH, Su CY, Yao YC, Cheng HC, Hsu TI, Wang PS, Su WP, Yang CJ, et al: Huntingtin-interacting protein-1 is an early-stage prognostic biomarker of lung adenocarcinoma and suppresses metastasis via AKT-mediated epithelial-mesenchymal transition. Am J Respir Crit Care Med 193: 869-880, 2016.

36. Attoub S, Arafat K, Hammadi NK, Mester J and Gaben AM: AKT2 knock-down reveals its contribution to human lung cancer cell proliferation, growth, motility, invasion and endothelial cell tube formation. Sci Rep 5: 12759, 2015.

37. Zhang Y, Liu S, Wang L, Wu Y, Hao J, Wang Z, Lu W, Wang XA, Zhang F, Cao Y, et al: A novel PI3K/AKT signaling axis mediates Nectin-4-induced gallbladder cancer cell proliferation, metastasis and tumor growth. Cancer Lett 375: 179-189, 2016.

38. Liu J, Chang F, Li F, Fu H, Wang J, Zhang S, Zhao J and Yin D: Palmitate promotes autophagy and apoptosis through ROS-dependent JNK and p38 MAPK. Biochem Biophys Res Commun 463: 262-267, 2015.
39. Ince S, Avdatek F, Demirel HH, Arslan-Acaroz D, Goksel E and Kucukkurt I: Ameliorative effect of polydatin on oxidative stress-mediated testicular damage by chronic arsenic exposure in rats. Andrologia 48: 518-524, 2016.

40. Ponugoti B, Dong G and Graves DT: Role of forkhead transcription factors in diabetes-induced oxidative stress, Exp. Diabetes Res 2012: 939751, 2012.

41. Du M, Wang Q, Li W, Ma X, Wu L, Guo F, Zhao S, Huang F, Wang $\mathrm{H}$ and Qin G: Overexpression of FOXO1 ameliorates the podocyte epithelial-mesenchymal transition induced by high glucose in vitro and in vivo. Biochem Biophys Res Commun 471: 416-422, 2016.

42. Duan S, Huang W, Liu X, Liu X, Chen N, Xu Q, Hu Y, Song W and Zhou J: IMPDH 2 promotes colorectal cancer progression through activation of the PI3K/AKT/mTOR and PI3K/AKT/FOXO1 signaling pathways. J Exp Clin Cancer Res 37: 304, 2017.

43. Jiang G, Huang C, Li J, Huang H, Jin H, Zhu J, Wu XR and Huang C: Role of STAT3 and FOXO1 in the divergent therapeutic responses of non-metastatic and metastatic bladder cancer cells to miR-145. Mol Cancer Ther 16: 924-935, 2017.

(i) (9) This work is licensed under a Creative Commons Attribution-NonCommercial-NoDerivatives 4.0 International (CC BY-NC-ND 4.0) License. 